\title{
Structural \& Functional Consequences of Chronic Psychosocial Stress on the Microbiome \& Host
}

Aadil Bharwani1 ${ }^{1,6}$, M. Firoz Mian6, Jane A. Foster 3 ,6, Michael G. Surette ${ }^{2,4}$, John Bienenstock ${ }^{1,6}$, \& Paul Forsythe 2, 5, 6

1Department of Pathology \& Molecular Medicine, McMaster University, 1280 Main Street West | Hamilton, Ontario, L8S 4L8, Canada

2Department of Medicine, McMaster University, 1280 Main Street West Hamilton, Ontario, L8S 4K1, Canada

3Department of Psychiatry \& Behavioral Neurosciences, McMaster University, 1280 Main Street West, Hamilton, Ontario L8S 4L8

${ }^{4}$ Farncombe Family Digestive Health Research Institute, 1280 Main Street West, Hamilton, Ontario L8S 4L8, Canada

${ }^{5}$ Firestone Institute for Respiratory Health, St. Joseph's Healthcare, 50 Charlton Avenue East Hamilton, Ontario, L8N 4A6, Canada

6McMaster Brain-Body Institute, St. Joseph's Healthcare, 50 Charlton Avenue East Hamilton, Ontario, L8N 4A6, Canada

Running title: Chronic Stress Effects on Microbiome and Host

\section{Address for correspondence}

Paul Forsythe, PhD.

McMaster University, Department of Medicine, The Brain-Body Institute, St. Joseph's Healthcare 50 Charlton Avenue East, T3302 Hamilton, Ontario L8N 4A6

Tel: 905-522-1155 ex 35890

Fax: 905-540-6593

E-mail: forsytp@mcmaster.ca 


\section{Aadil Bharwani}

\section{Abstract}

\section{Introduction}

Given the lasting impact of psychological distress on behavior, along with the role of the microbiome in neurobehavioral development, we sought to examine the relationship between the microbiota and stress-induced behavioral deficits.

\section{Methods}

Male C57BL/6 mice exposed to chronic social defeat were subjected to behavioral analysis and profiling of the intestinal microbiome. Mice were also analyzed for phenotypic and functional immune changes. A computational approach on 16S rRNA marker gene sequences was used to predict functional changes in the metagenome as a consequence of structural shifts in the microbiota.

\section{Results}

Chronic social defeat induced behavioral changes that were associated with reduced richness and diversity of the gut microbial community, along with distinct shifts at the level of operational taxonomic units (OTU) across phyla. The degree of deficits in social, but not exploratory behavior was correlated with group differences between the microbial community profile. In silico analysis predicted a shift in the functional profile of the microbiome: defeated mice exhibited reduced functional diversity and a lower prevalence of pathways involved in the synthesis and metabolism of neurotransmitter precursors and short-chain fatty acids. Defeated mice also exhibited sustained alterations in dendritic cell activation, and transiently elevated levels of IL-10+ T regulatory cells that were suppressed over time.

\section{Conclusions}

This study indicates that stress-induced disruptions in neurologic function are associated with altered immunoregulatory responses and complex OTU-level shifts in the microbiota. It is thus suggested that a dysbiotic state, along with specific changes in microbial markers, may predict the 


\section{Aadil Bharwani}

onset of adverse neurocognitive deficits commonly observed following exposure to severe stressors. The data also predict novel pathways that might underlie microbiota-mediated effects on brain and behavior, thus presenting targets for investigations into mechanisms and potential therapy.

Keywords: Psychosocial stress, gut microbiota, behavior, immune regulation, functional metagenome, chronic social defeat 


\section{Aadil Bharwani}

\section{Introduction}

There is abundant evidence demonstrating the adverse impact of stress on physiology and neurocognitive correlates during development and adulthood: trauma or abuse during early life increase the risk of psychiatric conditions and can impair the development of the stress response (Heim et al., 2008), while inadequate coping behavior contributes to the etiology of diseases such as gastrointestinal disorders and increased risk of depression (Dinan, 2005; Mayer, 2000). Amidst the efforts to elucidate the mechanisms underpinning this association, there has been a growing recognition of the importance of the microbiota to normal development and function of several physiological processes, including metabolism, immunity, and behavior. Indeed, so integral are these symbionts to host function that it has been suggested that almost all animals, including humans, should be viewed as multi-species organisms or "holobionts" (Gilbert et al., 2012).

The body of work demonstrating the systemic role of the microbiome, especially in neural development and function, is extensive. Disruption or absence of the microbiome impairs behavior and its development, leading to increased exploration, decreased apprehension, and impaired social behavior (Bercik et al., 2011; Desbonnet et al., 2014). Conversely, chronic administration of Lactobacillus rhamnosus (JB-1) alters GABAR expression in the brain, and reduces anxiety-like and depressive behavior (Bravo et al., 2011). Particularly, there is compelling evidence of bidirectional interaction between stress and the microbiome. Exposure to stress alters the structural composition of the intestinal microbiota (Bailey et al., 2011; O’Mahony et al., 2009), while germ-free (GF) status and intestinal colonization alter the developmental trajectory of the stress response (Neufeld et al., 2011; Sudo et al., 2004). Within the context of the holobiont paradigm, the influence exerted by these microorganisms on brain development and behavior is a consequence of the evolution of a multi-species organism. To provide clearer insight into the implications of the concept of the collective "self" for health and disease, and understand the functional relationship between the microbiota and stress-induced alterations, we need greater insight into the 


\section{Aadil Bharwani}

mechanisms, pathways, and consequences of communication along the microbiota-gut-brain axis.

Here, an anthropomorphic model is used to examine the impact of psychosocial stress on host-microbiota interactions, and the relationship between the microbiome and stress-induced behavioral deficits. We profile the community structure and species-level shifts in the intestinal microbiota, including in the relative abundance of Akkermancia muciniphilia and Coriobacteriaceae. These specific taxa have been previously reported to be associated with healthy and stress-exposed microbiome communities respectively (Bendtsen et al., 2012; Everard et al., 2013). Accordingly, in an effort to investigate the use of specific microbial community markers to predict adverse consequences on the host, we examined whether such alterations are retained, amidst broader shifts in the microbiota, across experimental studies. We also examine the nature of the stressinduced dysbiosis—specifically, the Firmicutes/Bacteroidetes ratio, which signals the status of the human gut microbiota in models of obesity and antibiotic-induced dysbiosis (Mariat et al., 2009; Sanderson et al., 2006; Thompson et al., 2015).

Given evidence of immune-mediated signaling along the microbiota-gut-brain axis in the literature (Desbonnet et al., 2010; Forsythe et al., 2010), we profile the immunoregulatory and innate immune phenotype, as well as the function of the peripheral immune system. Moreover, using a computational approach, we address the biological pathways that may be driving the effects of the microbiome on brain and behavior by profiling the predicted functional implications of structural shifts in the microbiome.

\section{Methods}

2.1 Animals. Male C57BL/6 mice, eight-weeks old, and male CD-1 mice, retired breeders, were acquired from Charles River (Montreal, QC, Canada). All animals were allowed to acclimatize to the housing facility for seven days prior to beginning the experiment. Animals were housed in standard conditions (12-h light-dark (LD) cycle, lights on at 07:00) with ad libitum access to standard rodent 
Aadil Bharwani

chow and water. All experiments followed the guidelines of the Canadian Council on Animal Care and were approved by the McMaster Animal Research Ethics Board.

2.2 Social Defeat. Chronic Social Defeat (CSD) procedures were conducted as previously described (Berton et al., 2006). Defeats were conducted over the course of 10 consecutive days. During each defeat session, intruder C57BL/6 mice were allowed to interact for 5-10 minutes with a novel resident CD-1 mouse. Intruder mice were carefully observed to ensure the demonstration of subordinate posturing. For 24 hours after each defeat session, mice were house in the same cage across a perforated Plexiglas divider to enable the transmission of visual and olfactory cues. Control mice were housed two per cage on either side of a Plexiglas divider while preventing any physical contact.

\subsection{Behavioral Testing}

2.3.1 Open Field Test (OFT). Testing was carried out in the dark phase of the LD cycle under dimlight conditions, one day after exposure to the final defeat session (Fig S1). After a one-hour habituation period in the testing room, mice were singly placed into an 18 x $38 \mathrm{~cm}$ clear Plexiglas enclosure for a period of 30 minutes. Total distance traveled, rearing count, and time spent in the center of the field were recorded via photo beam sensors outfitted around the arena (Motor Monitor; Kinder Scientific). The equipment was cleaned between each test.

2.3.2 Three-Chambered Sociability Test. All tests were conducted 2 days after the final defeat session, during the light phase of the LD cycle following a 30-minute habituation period in the testing room. The testing apparatus was a three-chambered Plexiglas box, with each chamber possessing the dimensions $24.5 \mathrm{~cm} \mathrm{~L} \mathrm{x} 44 \mathrm{~cm} \mathrm{~W}$ x $30 \mathrm{~cm} \mathrm{H}$. The dividing walls of the chambers possessed small openings that allowed mice access to each chamber. During the habituation phase of the test, a single test mouse was placed in the center chamber-with access to side chambers obstructed-and allowed to freely explore for five minutes. Following this, an unfamiliar sex- and strain-matched conspecific (stranger) was placed within a round, wire cup in one of the side 


\section{Aadil Bharwani}

chambers. An identical inverted wire cup was placed in the other side chamber. During the sociability phase, the test mouse was placed in the center chamber and allowed to freely explore all three chambers for a period of ten minutes. During each phase, distance moved, time spent in each chamber, and time spent in within-chamber zones were recorded by a video camera positioned directly over the testing apparatus (EthoVision XT; Noldus). Sociability scores were calculated using (time spent in mouse-chamber interaction zone/time spent in empty-chamber interaction zone). The equipment was cleaned and wiped down between test mice.

2.3.3 Aggressor Interaction Test. Following completion of the three-chambered sociability test, mice were placed for 10 minutes in a $24.5 \mathrm{~cm}$ x $44 \mathrm{~cm}$ arena. A novel aggressor CD-1 was placed under a round, wired cage at one end of the arena. Time spent in the aggressor interaction zone and non-interaction zone was calculated for each mouse (EthoVision XT; Noldus).

2.3.4 Light-Dark (LD) Test. In a separate cohort of mice, on day 3 following the final defeat session, testing was carried out in the dark phase of the LD cycle under dim-light conditions. After a onehour habituation period in the testing room, mice were singly placed into an 18 x $38 \mathrm{~cm}$ clear Plexiglas enclosure containing a black insert at one end for a period of 10 minutes. Kinderscientific Motor Monitor software was used to record time spent in the light zone and number of entries into the light zone. The equipment was cleaned and wiped down between test mice.

2.4 Tissue Collection and Splenocyte Isolation. 5 days after the final defeat session, following completion of all behavioral tests, or 17 days after the final defeat session - due to an opportunity afforded by the use of these mice in a separate, independent experiment-mice were euthanized and trunk blood was collected for serum analysis. Spleens were harvested 5 or 17 days after the final defeat session and dispersed using a cell strainer in cold, sterile PBS. Cell suspensions were centrifuged at $1500 \mathrm{rpm}$ for 10 minutes at $4^{\circ} \mathrm{C}$, then re-suspended in RBC lysis buffer for $1-2$ minutes. The resulting solution was centrifuged before cell pellets were washed with $5 \mathrm{ml}$ of complete RPMI 1640 medium: 10\% fetal bovine serum, penicillin/streptomycin antibiotics, 2mM L- 
Aadil Bharwani

glutamine, and $0.01 \% \beta$-mercaptoethanol. Viable cell numbers were assessed by Trypan Blue exclusion and diluted in RPMI to a concentration of $10^{7}$ cells $/ \mathrm{ml}$.

2.5 Flow Cytometry. Splenocytes $\left(10^{6}\right)$ were stained for markers of dendritic cell (DC) maturation and function-CD11c-APC-Cy7, MHCII-PE-Cy7, CD80-PerCP-Cy5, CD86-APC-or regulatory T cells-CD3-APC, CD4-FITC, CD25-PE-Cy7, intracellular Foxp3- PerCP-Cy5, intracellular IL-10-PE (BD Pharmingen, San Diego, CA, USA; eBiosciences, San Diego, CA, USA). Following surface staining, cells were fixed and permeabilized with BD Cytofix/cytosperm before staining for intracellular markers. Data were acquired with FACSCanto (Becton Dickinson, Oakville, ON, Canada) and analyzed using FlowJo (TreeStar, Ashland, OR, USA).

2.6 In vitro Splenocyte Stimulation. For anti-CD3/CD28 stimulation, splenocytes $\left(1 \times 10^{6}\right)$ were incubated in a 96-well cell culture plate coated with anti-CD3 antibody $(2 \mu \mathrm{g} / \mathrm{ml})$ and in the presence of anti-CD28 antibody $(2 \mu \mathrm{g} / \mathrm{ml})$. For stimulation with lipopolysaccharide (LPS), isolated splenocytes were incubated for 48 hours at $37^{\circ} \mathrm{C}$ with $5 \% \mathrm{CO} 2$, following which they were stimulated with $20 \mu \mathrm{L}$ of $10 \mu \mathrm{g} / \mathrm{ml}$ of LPS. After incubation for a total of 72 hours, all samples were centrifuged at $1500 \mathrm{rpm}$ for 10 minutes at $4^{\circ} \mathrm{C}$. Supernatants were isolated and stored at $-20^{\circ} \mathrm{C}$ for cytokine analyses (eBiosciences, San Diego, CA, USA).

2.7 16s rRNA Analysis of Bacterial Composition. One day before the first defeat session and one day following the final defeat session, fecal pellets were collected and stored at $-80^{\circ} \mathrm{C}$ for molecular analysis of microbiota. DNA extraction was carried out using a previously described protocol that enhances DNA recovery from microbial communities (Sibley et al., 2011) with modifications (Whelan et al., 2014) to increase quantitative recovery of bacteria across different taxa. Quantitative PCR (qPCR) was used to measure total eubacterial load using the universal bacterial primers 8fM and Bact515R (Nadkarni et al., 2002). Bacterial community profiling of 16S rRNA gene was carried out using a modified bar coded Illumina sequencing method (Bartram et al., 2011). Paired end reads of the V3 region were performed using the 341F and 518R primers (Muyzer et al., 


\section{Aadil Bharwani}

1993). $250 \mathrm{nt}$ paired-end sequencing was carried out on a MiSeq Illumina sequencer as per manufacturer's instructions. This approach provided overlapping sequence reads of the V3 region, which could be used for correcting poor quality base calls and increasing sequencing accuracy. Sequencing was carried out on a MiSeq Illumina sequencer in the McMaster Genome Center (McMaster University).

The MiSeq data was processed by an in-house bioinformatics pipeline (Whelan et al., 2014) that incorporates quality filtering. Sequencing results produced 7458 operational taxonomic units (OTUs), and a minimum, maximum, and median of 5800, 282124, and 206300.5 reads/sample respectively. Using QIIME (Caporaso et al., 2010), singletons were excluded and OTU tables underwent ten repeated rarefactions at multiple sequencing depths to enable equal reads across samples. For alpha diversity analysis, Chao1 and Phylogenetic Diversity metrics were recruited using the alpha diversity workflow script. Each metric was implemented using the same number of sequences as the most indigent sample (5800). Mann Whitney $U$ tests were used to assess statistical significance of measures derived from alpha diversity metrics. For beta-diversity analyses, Jackknife resampling at a sequencing depth equal to $80 \%$ of the most indigent sample was used to generate weighted and unweighted unifrac distance matrices. Between-group (dis)similarity was assessed using the Monte Carlo Permutation Procedure (MCPP) (999 permutations) and the analysis of similarities (ANOSIM) test. The Mann-Whitney U test was used to examine the abundance of Akkermancia and Coriobacteriaceae-groups predicted a priori to be altered following chronic stress. The G-test of goodness-of-fit and the Benjamini Hochberg correction for multiple comparisons (False Discovery Rate $<0.1$ ) was used to analyze differential abundance of OTUs in either group using data first rarefied to a sequencing depth of 5800 and then filtered to eliminate OTUs observed fewer than 10 instances. To analyze the association between microbiome and behavior, Procrustes analysis—-which compares and transforms distance matrices through rotation, scaling, and translation to minimize the distance between corresponding points (Gower, 
Aadil Bharwani

1975) — and the mantel test were performed on distance matrices for unweighted unifrac and behavioral data that distinguished between control and defeated groups: aggressor interaction and rearing behavior.

2.8 In silico Metagenomics. Predictions regarding the functional composition of the microbiome were made on 16S rRNA-derived OTUs using a computation approach: phylogenetic investigation of communities using reconstruction of unobserved states (PICRUSt) (Langille et al., 2013). OTU abundances were normalized to known or predicted $16 \mathrm{~S}$ copy number abundances. Using KEGG pathway metadata, KEGG orthologs (KO) were categorized by their function to level 3 of the pathway hierarchy. Group differences in functional diversity were calculated using the Shannon Index and analyzed using the Mann Whitney U test. PCoA plots were created using Bray-Curtis distances with the QIIME v1.8.0 software suite (Caporaso et al., 2010), and differences between the functional profiles of control and defeated groups were analyzed with the ANOSIM test. Differential frequency of functionally categorized gene counts was analyzed using the G-test of goodness-of-fit and the Benjamini Hochberg correction for multiple comparisons (FDR < 0.05).

2.9 Statistical Analysis. Behavioral and immunological data were analyzed in GraphPad Prism 5 using two-tailed students $t$ test, Mann-Whitney U test, or ANOVA, with post-hoc tests that utilized Bonferroni corrections. Effect size is reported for p-values near statistical significance using probability of superiority. Results in figures are expressed as mean \pm SEM. Statistical significance is denoted as $*(\mathrm{P}<0.05),{ }^{* *}(\mathrm{P}<0.01)$, and ${ }^{* * *}(\mathrm{P}<0.001)$.

\section{Results}

\subsection{Exposure to chronic social defeat induces deficits in social and exploratory behavior}

In order to characterize changes in the behavioral phenotype following exposure to chronic stress, animals were subjected to measures that evaluate social, exploratory, and anxiety-like behaviors. Socially defeated mice exhibited pronounced avoidance of a novel CD1 aggressor mouse 


\section{Aadil Bharwani}

during the aggressor interaction test, opting to spend the majority of the time in the non-interaction zone of the field (Fig. 1A; Welch-corrected $t=15.74, \mathrm{df}=27, P<0.0001)$. Defeated mice also exhibited marked deficits in social preference during the three-chambered test. Unlike unstressed control mice, which spent more time exploring the mouse chamber, defeated mice spent significantly more time exploring the empty chamber than the chamber containing the sex-, and strain-matched conspecific mouse (Fig. 1B; Group x chamber interaction, $F_{2,66}=27.98, P<0.0001$; Bonferroni-corrected post hoc test, empty versus mouse chamber for defeated group, $t=4.36, P<$ 0.001). Although the defeated group also exhibited lower activity levels during the habituation phase (distance traveled, $\mathrm{t}=3.045, \mathrm{df}=33, P=0.0046$ ), this is unlikely to have contributed to differences in sociability, given that sociability scores-a measure of relative time spent interacting with the mouse or object in the respective chambers-were also lower in the defeated group (Fig. $1 C ; t=3.60, \mathrm{df}=33, P=0.001$ ), demonstrating that defeated mice spent less time interacting with the novel conspecific.

Chronic exposure to social defeat also altered behavior during the OFT. Defeated mice exhibited reduced locomotion $(t=2.46, \mathrm{df}=33, P=0.019)$ and consistently lower levels of exploratory behavior, as evidenced by fewer rearing counts across all time intervals (Fig. 1D; main effect of Group, $F_{1,165}=83.14, P<0.0001$; Group $\mathrm{x}$ time interaction, $F_{5,165}=3.59, P=0.0042$; Bonferroni-corrected post hoc test, control versus defeated group, $P<0.001$ for all six time intervals). Anxiety-like behavior on the OFT remained unaffected, as demonstrated by the lack of difference in time spent in the center of the field (Fig. 1E; $P=0.5550$ ). However, given previous evidence of the effect of chronic stress on anxiety (Kinsey et al., 2007), anxiety-like behavior was further evaluated in a separate cohort of mice using the LD test. Similar to previously, defeated mice exhibited reduced rearing behavior $(t=5.309, \mathrm{df}=15, P<0.0001)$, but not time spent in the center $(t=2.021, \mathrm{df}=15, P=0.0615$, probability of superiority= 0.757$)$ on the OFT. On the LD test, there was no statistical difference in time spent in the light zone (Fig. S2A; $t=0.1209, \mathrm{df}=14, P=0.9055$ ); 
Aadil Bharwani

however, defeated mice made fewer entries into the light zone (Fig. S2B; $t=2.545, \mathrm{df}=14, P=$ 0.0234), suggesting increased anxiety-like behavior.

\subsection{Exposure to chronic social defeat alters the microbiome structure}

To explore the functional relationship between stressor-induced changes in the microbiome and behavior, we first sought to profile the structural composition of the microbiota in mice exposed to prolonged social stress. Alpha-diversity analysis of fecal samples revealed differences within the composition of the microbial community of each group. As visualized in the rarefaction curves (Fig. 2A), there was a pronounced decrease in the overall diversity of the microbiome following exposure to stress. In contrast, the microbial community in the control group remained relatively stable at both time points and boasted greater species diversity than that of socially defeated mice (Faith's PD, Mann-Whitney $U=17, P=0.04$ ). Chronic stress also reduced the richness of the community, resulting in a lower estimate of rare species or OTU classes (Fig. 2B; Chao1, Mann-Whitney $U=15, P=0.0244)$.

Examination of the microbiome profile revealed clustering of the gut microbiota by stress exposure group (Fig. 2C). Analysis of unweighted Unifrac PCoA plots using the MCPP revealed shorter distances between intra-group samples than samples between groups (999 permutations, non-parametric $P=0.018$ ). These results were confirmed using the ANOSIM test on unweighted Unifrac distances, which revealed group differences in the microbial communities (999 permutations, $P=0.001$ ). That there was no clustering of samples using weighted Unifrac distances (MCPP, 999 permutations, non-parametric $P=0.256$ ) suggests that profile differences are driven by changes at the OTU-level of the community rather than shifts in the relative abundance of the taxa.

To examine the sensitivity of certain microbial groups to stress exposure and further explore changes in the community, we examined the abundance of species predicted, $a$ priori, to be altered in the defeated group. Defeated mice exhibited a trend towards lowered fecal levels of Akkermansia (species: unclassified) (Mann Whitney $U=20, P=0.0642$, probability of superiority= 


\section{Aadil Bharwani}

0.803) and significantly lower levels of Coriobacteriaceae (genus: other) than the control group (0.0635\% versus $0.142 \%$, Mann Whitney $U=12, P=0.0418)$.

Previous studies have demonstrated dysbiotic states to be associated with a shift in dominant taxa at the phylum level (Mariat et al., 2009; Sanderson et al., 2006; Thompson et al., 2015). Thus, we examined the phylogenetic distribution of OTUs in the control and defeated microbial communities. The relative abundances of the major phyla were relatively similar between the two groups (Fig. 3A; Firmicutes, control versus defeated groups: 36.96\% versus 39.16\%; Bacteroidetes, control versus defeated groups: $60.32 \%$ versus $58.26 \%$ ), revealing the absence of a straightforward shift in the structure of the community. Initial group differences however, were revealed upon analysis of distribution at the class level (Fig. 3A; Bacilli, control versus defeated groups: $12.06 \%$ versus $20.26 \%$; Clostridia, control versus defeated groups: $24.4 \%$ versus $18.28 \%$ ). The G-test of goodness-of-fit was implemented in QIIME (Caporaso et al., 2010) to investigate differential abundance of individual OTUs between the two communities (FDR $<0.1$ ). At this criterion, 25 OTUs were differentially represented between the two groups (Fig. S3); 23 of these OTUs belonged to either of the two major phyla (Fig. 3B). In the microbial community of defeated mice, the abundance of distinct OTUs belonging to Firmicutes and Bacteroidetes were either increased ( 8 members of Firmicutes) or decreased ( 6 members of Firmicutes, 9 members of Bacteroidetes), demonstrating complex structural alterations following chronic exposure to social defeat.

To examine the relationship between changes in the microbiome and behavioral deficits, Procrustes analysis was used to compare the unweighted Unifrac matrix to a distance matrix of behavior on the aggressor interaction test (Fig. 3C). The data exhibited a statistically significant fit between the two matrices, based on 10000 Monte Carlo iterations $\left(\mathrm{M}^{2}=0.786, P=0.0052\right)$. This relationship was further evidenced by a statistically significant correlation between the two variables (Mantel $r=0.167, P=0.029,999$ permutations) and appeared to be specific to social 
Aadil Bharwani

avoidance behavior, as no such association was observed between the unweighted Unifrac data and rearing behavior $\left(\mathrm{M}^{2}=0.848, P=0.056\right)$.

\subsection{Chronic social defeat induces lasting changes in immunoregulatory responses}

Given previous evidence of immune-mediated communication along the microbiota-gutbrain axis (Desbonnet et al., 2010; Forsythe et al., 2010), we investigated changes in immune phenotype and function as a consequence of exposure to social defeat.

Chronic stress altered immune function, with defeated mice exhibiting elevated serum IL-6 levels, five days after the final defeat session (Fig. 4A; $t=3.837, \mathrm{df}=15, P=0.0016$; Control group= $0.0838 \pm 0.0838 \mathrm{pg} / \mathrm{ml}$, Defeated group $=44.52 \pm 14.04 \mathrm{pg} / \mathrm{ml})$. No significant differences were observed in serum levels of IL-10 $(P=0.327$; Control group $=2.610 \pm 0.2891 \mathrm{pg} / \mathrm{ml}$, Defeated group= $3.188 \pm 0.5522 \mathrm{pg} / \mathrm{ml})$, IL-17 $(P=0.0652$; Control group= $1.691 \pm 0.811 \mathrm{pg} / \mathrm{ml}$, Defeated group= $5.585 \pm 2.099 \mathrm{pg} / \mathrm{ml}$, probability of superiority= 0.703$)$, IL-4 $(P=0.1120$; Control group= $0.5000 \pm$ $0.0099 \mathrm{pg} / \mathrm{ml}$, Defeated group= $0.7663 \pm 0.1216 \mathrm{pg} / \mathrm{ml}$ ), or macrophage inflammatory protein (MIP)-2 $(P=0.9151$; Control group $=21.53 \pm 2.880 \mathrm{pg} / \mathrm{ml}$, Defeated group $=21.01 \pm 4.046$ $\mathrm{pg} / \mathrm{ml}$ ).Changes in IL-6 were transient, as serum IL-6 levels were no longer distinguishable between the two groups seventeen days following defeat $(P=0.8020$; Control group $=4.200 \pm 0.3544$ $\mathrm{pg} / \mathrm{ml}$, Defeated group $=3.967 \pm 0.8442 \mathrm{pg} / \mathrm{ml}$ )

To investigate whether such changes were due to immunoregulatory alterations, we characterized the phenotype of regulatory T cells and dendritic cells. There were no significant differences in the number of splenocytes isolated from either group $(P=0.2705$; Control group= $101.0 \pm 9.648 \times 10^{6}$ cells, Defeated group $=128.1 \pm 23.82 \times 10^{6}$ cells). Social defeat did result in sustained changes in phenotype of T cells and dendritic cells. While there were no differences in the population of Foxp3-expressing CD4+CD25+ T cells at $5(P=0.6409)$ or 17 days $(P=0.0724)$ after stress exposure, there were distinct differences in the IL-10-expressing CD4+ CD25+ 


\section{Aadil Bharwani}

population over time. Chronic social defeated increased IL-10+CD4+ CD25+ T cells in the spleens of defeated animals 5 day post-stressor (Fig. $4 \mathrm{~B} ; t=2.748, \mathrm{df}=8, P=0.0252$ ), but led to a decrease in levels of this cell population 17 days after stress exposure (Fig. 4C; $t=3.109$, $\mathrm{df}=16, P=0.0067$ ). Given this, the supernatant from splenocytes isolated 5 or 17 days after the final defeat session was analyzed to examine whether the functional changes paralleled phenotypic alterations. At five days following the final stressor, there were no differences in the release of IL-10 from anti-CD3/CD28 antibody-stimulated splenocytes $(P=0.5007$; Control group $=2019 \pm 332.1 \mathrm{pg} / \mathrm{ml}$, Defeated group= $2489 \pm 578.0 \mathrm{pg} / \mathrm{ml}$ ). At seventeen days following chronic social defeat, there was a trend towards reduced IL-10 release from splenocytes of defeated mice following anti-CD3/CD28 antibody stimulation (Fig. 4D; $t=2.020, \mathrm{df}=16, P=0.0605$, probability of superiority= 0.753 ), but not LPS stimulation $(P=0.353$; Control group $=1577 \pm 225.1 \mathrm{pg} / \mathrm{ml}$, Defeated group $=1999 \pm 406.7 \mathrm{pg} / \mathrm{ml})$. In contrast, there was no difference in the release of TNF by splenocytes stimulated with either anti-CD3/CD28 antibodies $(P=0.2430$; Control group= $1904 \pm 411.0 \mathrm{pg} / \mathrm{ml}$, Defeated group= 1283 $\pm 247.7 \mathrm{pg} / \mathrm{ml})$ or LPS $(P=0.8144$; Control group $=9727 \pm 476.6 \mathrm{pg} / \mathrm{ml}$, Defeated group $=9450 \pm$ $1157 \mathrm{pg} / \mathrm{ml}$ ) at seventeen days following CSD.

To investigate whether immunoregulatory alterations were associated with phenotypic changes in innate immune activation, dendritic cells were examined for markers of maturation and activation. Analysis revealed that following chronic stress exposure, levels of CD86 and MHCII expression on CD11c+ DCs at both 5 and 17 days post-stressor remain unchanged (CD86: 5 days post-stressor, $P=0.1609 ; 17$ days post-stressor, $P=0.9750$; MHCII: 5 days post-stressor, $P=0.7181$; 17 days post-stressor, $P=0.405)$. The levels of the co-stimulatory molecule CD80 however, were enhanced at both 5 days (Fig. $4 \mathrm{E} ; t=2.956 \mathrm{df}=8, P=0.0183$ ) and 17 days post-stressor (Fig. 4F; $t=$ $3.268 \mathrm{df}=16, P=0.0048)$, suggesting enhanced $\mathrm{DC}$ activation. 
Aadil Bharwani

\subsection{Predictive analysis reveals altered functional microbiota profile following social defeat}

To investigate the implications of structural changes in the microbiome, functional predictions were made on 16S rRNA-derived OTUs using PICRUSt, a computational method (Langille et al., 2013). Analysis using the Shannon Index revealed reduced functional diversity of KEGG pathways in the microbial community of defeated mice (Fig. $5 \mathrm{~A} ; t=2.218, \mathrm{df}=16, P=0.0413$ ). PCoA plots of functional KEGG pathways generated using Bray-Curtis distances exhibited clustering of samples according to exposure to social defeat (Fig. 5B), which was confirmed following statistical analysis (ANOSIM $R=0.1918, P=0.027,999$ permutations). Upon further investigation, a total of 145 KEGG pathways were found to be differentially represented between control and defeated groups (FDR < 0.05), of which a subset are displayed in Table 1. Predictive analysis indicated in the defeated group a lower frequency of pathways involved in fatty acid metabolism and biosynthesis, including the metabolism of propanoate and butanoate-conjugate bases of the short chain fatty acids (SCFAs), propionic and butyric acid. Defeated mice also exhibited reduced abundance of pathways involved in the biosynthesis and metabolism of tyrosine and tryptophan: molecules that serve as precursors for the synthesis of dopamine (DA), norepinephrine, serotonin (5-HT), and melatonin respectively.

\section{Discussion}

In this study, we offer a structural and functional analysis of the consequences of chronic social defeat stress the murine microbiome and the host. We demonstrate that psychosocial stress induces complex and nuanced OTU-level shifts in the structural composition of the intestinal microbiota, including changes in the relative abundance of microbial groups that are sensitive to the state of the host (Bendtsen et al., 2012; Everard et al., 2013); these shifts are associated with the anthropomorphic neurocognitive and immunoregulatory disruptions commonly observed in both animal models and patients with affective conditions (American Psychiatric Association, 2013; 


\section{Aadil Bharwani}

Maes, 1995). We also frame these structural changes in the microbiome within the context of the associated functional consequences on the metagenome in order to predict the biological pathways that may underlie the effects of the microbiota on brain and behavior: social defeat reduces the prevalence of synthesis and metabolism pathways for short-chain fatty acids and neurotransmitter precursors.

Following chronic social stress exposure, defeated mice exhibited a distinct intestinal microbiota profile from that of the control group. Previous studies have also demonstrated an effect of stress on the microbiome profile, including a decrease in Bacteroides and an increase in the Clostridium genus (Bailey et al., 2011; O’Mahony et al., 2009) However, little is known about the nature of these structural alterations at the species membership level, the functional implications associated with the resulting dysbiosis, and its relationship with stress-induced behavioral deficits. Studies investigating obesity and environmental stress-induced dysbiosis of the gut microbiome, such as that produced by antibiotic administration, have demonstrated a binary shift at the phylum level in the Firmicutes/Bacteroidetes ratio-a measure indicative of the normal healthy status of the human intestinal microbiome (Mariat et al., 2009; Sanderson et al., 2006; Thompson et al., 2015) Here however, in addition to decreasing the overall richness and diversity of the community, chronic stress engendered a more complex form of dysbiosis. Rather than a phylum-level binary shift in the community, we observed both increases and decreases in the frequency of distinct OTUs in the major phyla that comprise the microbial population. While not reaching statistical significance, defeated mice also exhibited lowered relative abundance of Akkermansia-a taxon whose relative abundance in the microbiota is associated with healthier states (Everard et al., 2013) .Furthermore, in contrast to a previous study demonstrating higher levels of Coriobacteriaceae following exposure to a grid floor model of stress in Balb/c mice (Bendtsen et al., 2012), social defeat led to reduced levels in stressed C57BL/6 mice. These observations demonstrate complex structural alterations and indicate the sensitivity of certain bacterial groups 


\section{Aadil Bharwani}

to stress exposure, although the precise magnitude and direction of the shift may vary between different mouse strains and/or models of stress. While our efforts here were limited to evaluating the microbiota community in fecal material, a recent study using the restraint stress model demonstrated the differential impact of stress on the composition of the luminal versus mucosaassociated colonic community (Galley et al., 2015). Only the diversity of the mucosa-associated community was impacted by the stressor. Furthermore, restraint stress induced compartmentspecific shifts in the community-wide profile of the colonic microbiota, as well as in the relative abundance of specific taxa such as Lactobacillus. These results emphasize the need for future studies to take into account compartment-specific niche differences in order to understand how such alterations might impact host health.

Exploring the role of these distinct microbiota alterations in stress-induced behavioral changes revealed an association between the microbiome profile and deficits in social behavior on the aggressor interaction test. That the association was limited to this particular test, where defeated mice avoided a novel stimulus resembling the original traumatic episodes, lends weight to the specific nature of this nexus. No such association was observed with deficits in exploratory behavior on the OFT. The relationship between the microbiota and social behavior has been demonstrated previously in GF mice, which display impairments in social cognition development, and in offspring from the maternal immune activation model of autism, which exhibit dysbiosis and deficits in social and communicative behavior,(Desbonnet et al., 2014; Hsiao et al., 2013). Indeed, some of these deficits are normalized through post-weaning colonization or treatment with $B$. fragilis. From a clinical perspective, aggressor avoidance following social defeat bears remarkable similarity to phobic avoidance behavior of trauma-related stimuli in individuals with posttraumatic stress disorder (American Psychiatric Association, 2013). These findings also parallel the comorbidity between stress-related psychiatric conditions and functional bowel disorders (Dinan et al., 2006; Mayer, 2000). Individuals exposed to severe wartime stress have a greater risk of 


\section{Aadil Bharwani}

irritable bowel syndrome (IBS), which is associated with alterations in the intestinal microbiota (Collins and Bercik, 2009; Klooker et al., 2009). Given the evidence provided here of the specific and nuanced nature of microbiota alterations, the sensitivity of certain microbial groups to stress exposure, and its association with stress-induced behavioral deficits, we propose that investigation into the use of microbial community markers to predict adverse neurocognitive consequences following exposure to severe stress is warranted.

Paralleling the suite of immunological changes observed in psychiatric conditions (Maes, 1995), exposure to social defeat induced alterations in immune profile. In addition to a transient increase in serum IL-6 there were long-term systemic changes at the cell population level. Spleens from defeated mice exhibited a greater population of activated dendritic cells at both 5 and 17 days following completion of the stress procedure, indicating sustained changes in innate immune activation. Defeated mice also exhibited alterations in the IL-10+CD4+CD25+ population, which is indicative of $\operatorname{Tr} 1$ regulatory T cells: a class of adaptive Tregs that produces high levels of the antiinflammatory/regulatory cytokine, IL-10, and has been previously demonstrated to prevent the development of experimental colitis (Groux et al., 1997). The increased population of IL-10+ CD4+CD25+ T cells at 5 days post-defeat may be reflective of a homeostatic response to counteract stress-induced inflammation (Boer et al., 2015). In contrast, at 17 days following cessation of the social defeat procedure, the defeated group exhibited reduced levels of this Treg population, indicating long-term alterations in the immunoregulatory profile-consistent with the adverse consequences of chronic stress. Furthermore, while differences did not reach statistical significance, stimulation of spleen-derived T cells from defeated animals at this time-point resulted in a trend towards reduced IL-10 release. The immune alterations also exhibited a distinct temporal profile: unlike phenotypic changes in DCs and the Tr1 cell population, which were evident at least seventeen days following the cessation of chronic stress, group differences in serum IL-6 levels were no longer evident by this time point. Overall, the immune data suggests that chronic social 


\section{Aadil Bharwani}

defeat stress induces long-term changes in immune regulation and priming of the innate immune response. Furthermore, given that we only evaluated acute changes in the microbiome and behavior (36 hours and days 1-2 following the final defeat session respectively), future studies are needed to provide insight into whether the observed changes are long lasting in order to investigate the extent of the impact of chronic stress on the adult microbiota and the associated behavioral deficits.

Although we cannot yet determine the direction of causality linking these immune alterations to behavioral and microbiome changes, our findings align with the critical role of the microbiota in priming the immune system (Clarke et al., 2010) and evidence implicating immune signaling in communication along the microbiota-gut-brain axis: the maternal separation stressinduced inflammatory profile induced and its associated behavioral deficits are both normalized upon administration of $B$. infantis (Desbonnet et al., 2010). Similarly, administration of $B$. infantis to human patients diagnosed with IBS induces immunomodulatory shifts in the cytokine levels of peripheral blood mononuclear cells (O’Mahony et al., 2005).

While the role of immune and vagal signaling routes have been demonstrated in the gutbrain communications infrastructure (Bravo et al., 2011; Perez-Burgos et al., 2014), there remains much to be investigated regarding the functional alterations following structural changes in the microbiome. In defeated mice, in silico metagenomic analysis predicted a lower frequency of functional pathways for the synthesis and metabolism of tyrosine and tryptophan-precursors to DA and 5-HT respectively. The role of the microbiota in regulating host 5-HT levels has been highlighted extensively: more than $90 \%$ of the total 5 -HT content is synthesized in the gut (Erspamer, 1966), while GF mice exhibit altered levels of 5-HT in the plasma and hippocampus (Clarke et al., 2012; Wikoff et al., 2009). These data indicate the presence of peripheral mechanisms through which indigenous species might influence the neurochemistry of the central nervous system (CNS). A recent study highlighted one such mechanism, which involved increased 5-HT 


\section{Aadil Bharwani}

synthesis by enterochromaffin cells through metabolites produced by colonic spore-forming bacteria (Yano et al., 2015). Interestingly, this population of microbes is predominantly comprised of Clostridia, which potently induce IL-10 expression in Tregs (Atarashi et al., 2013). Both observations are consistent with our results: defeated mice exhibited a lower abundance of Clostridial species (Fig. 3A) as well as reduced IL-10+ Tr1 Tregs (Fig. 4C), thus indicating a high degree of logical agreement between the predicted functional profile, our measured observations, and previous evidence in the literature. Given the vast array of receptors acted upon by 5-HT, including those expressed on peripheral neurons and immune cells (Baganz and Blakely, 2012), further research is needed to explore the implications of microbiota-dependent deficits in 5-HT signaling on stress-induced changes in behavior.

Our analyses also predicted reduced frequency of fatty acid biosynthesis and metabolism pathways, including that of propanoate and butanoate-the byproducts of dietary carbohydrate fermentation by intestinal microorganisms. Previous studies have extensively investigated the indirect neuroactive properties of these molecules. Butyrate is a potent histone deacetylase (HDAC) inhibitor that exerts antidepressant-like effects by increasing histone acetylation in the frontal cortex and hippocampus, and consequentially, raising Bdnf transcript levels (Schroeder et al., 2007). Through the SCFA receptor FFAR2, the microbiota also regulate microglia homeostasis in the CNS (Erny et al., 2015). Deficits in microglia maturation and function in GF mice were reversed by chronic administration of a SCFA mix comprising of butyrate, propionate, and acetate. Similarly, acetate influences appetitive and feeding behavior by inducing the expression of regulatory neuropeptides, via effects exerted through central mechanisms in the hypothalamus (Frost et al., 2014). Although it was previously unclear whether the systemic levels of these metabolites achieved in vivo were sufficient to produce behavioral changes, progress has been made by discovering their presence in the cerebrospinal fluid and the brain, and demonstrating that colonderived SCFAs cross the blood-brain barrier and preferentially accumulate in the hypothalamus, 


\section{Aadil Bharwani}

where they can affect CNS activity (Frost et al., 2014; Wyss et al., 2011). Such observations implicate functional changes in the microbiome as mediators of stress-induced changes in behavior. It should be emphasized that the in silico results are purely predictive in nature, and while potentially useful in directing future functional studies, there exist obvious limitations in such techniques. While studies have shown a high degree of correlation (0.8-0.9) between PICRUSt predictions and measured observations of gene content through metagenomic sequencing (Langille et al., 2013), the relationships between the metagenome and metabolite production are not straightforward. Thus caution must be taken not to over interpret the results of the PICRUSt analysis in the current study and the influence of chronic stress on microbial metabolism of both SCFA and tryptophan must be confirmed with in depth analysis of metagenomics and metabolic products. However, that the inferred changes in this study align with evidence in the literature does lend weight to these results and paves the way for targeted investigations into biological pathways that drive the effects of the microbiota on the host.

Although the literature is replete with evidence of microbiota-gut-brain communication, there exists a large gap in our understanding of the functional relationship between the microbiome and stress-induced changes in brain and behavior. The challenge lies in unraveling the mechanisms underlying the symbiotic microbiota-host relationships, and understanding how these relationships are altered upon exposure to chronic stress. In association with neurobehavioral disruptions that are analogous to those observed in psychiatric conditions, we demonstrate pronounced alterations in immune regulation, along with complex and nuanced shifts in the microbial community following chronic exposure to psychosocial stress. Using a computational approach, we address a critical gap in the literature by providing functional context to structural microbiota changes, thus enabling future research into the molecular mechanisms underlying signaling pathways along the microbiota-gut-brain axis. Furthermore, these findings emphasize the potential use of microbiota- 
Aadil Bharwani

based biomarkers and even novel therapeutic targets in and against the adverse psychiatric consequences of chronic stress. 
Aadil Bharwani

\section{Contributors}

P.F, J.B., \&. A.B conceptualized the study. P.F, J.B., A.B. \& J.A.F designed experiments. A.B. performed animal experiments. A.B. and M.F.M. prepared samples and carried out FACS analysis. M.G.S performed 16S rRNA DNA sequencing. A.B. acquired and analyzed the data and wrote the initial draft of the manuscript. A.B., M.F.M., J.A.F., M.G.S., J.B., \& P.F. contributed to data interpretation and revised the manuscript. All authors approved the final version of this article.

\section{Acknowledgments}

This project is funded by a grant from the Office of Naval Research (\#N00014-14-1-0787). A.B. gratefully acknowledges funding support from the Canadian Institutes of Health Research (GSM136180). P.F. is supported by a Career Award from the Department of Medicine, McMaster University. Equipment support was provided by funds from the Canadian Foundation for Innovation to J.A.F., M.G.S is supported as a Canada Research Chair. 


\section{Aadil Bharwani}

\section{References}

American Psychiatric Association, 2013. Diagnostic and statistical manual of mental disorders (5th ed.). American Psychiatric Association, Washington, DC.

Atarashi, K., Tanoue, T., Oshima, K., Suda, W., Nagano, Y., Nishikawa, H., Fukuda, S., Saito, T., Narushima, S., Hase, K., 2013. Treg induction by a rationally selected mixture of Clostridia strains from the human microbiota. Nature 500, 232-236.

Baganz, N.L., Blakely, R.D., 2012. A dialogue between the immune system and brain, spoken in the language of serotonin. ACS Chem. Neurosci. 4, 48-63.

Bailey, M.T., Dowd, S.E., Galley, J.D., Hufnagle, A.R., Rebecca, G., Lyte, M., 2011. Exposure to a Social Stressor Alters the Structure of the Intestinal Microbiota: Implications for Stressor-Induced Immunomodulation. Brain. Behav. Immun. 25, 397-407.

Bartram, A.K., Lynch, M.D.J., Stearns, J.C., Moreno-Hagelsieb, G., Neufeld, J.D., 2011. Generation of multimillion-sequence 16S rRNA gene libraries from complex microbial communities by assembling paired-end Illumina reads. Appl. Environ. Microbiol. 77, 3846-3852.

Bendtsen, K.M.B., Krych, L., Sørensen, D.B., Pang, W., Nielsen, D.S., Josefsen, K., Hansen, L.H., Sørensen, S.J., Hansen, A.K., 2012. Gut Microbiota Composition Is Correlated to Grid Floor Induced Stress and Behavior in the BALB/c Mouse. PLoS One 7, e46231.

Bercik, P., Denou, E., Collins, J., Jackson, W., Lu, J., Jury, J., Deng, Y., Blennerhassett, P., Macri, J., McCoy, K.D., Verdu, E.F., Collins, S.M., 2011. The intestinal microbiota affect central levels of brain-derived neurotropic factor and behavior in mice. Gastroenterology 141, 599-609.

Berton, O., Mcclung, C.A., Dileone, R.J., Krishnan, V., Renthal, W., Russo, S.J., Graham, D., Tsankova, N.M., Bolanos, C.A., Rios, M., Monteggia, L.M., Self, D.W., Nestler, E.J., 2006. Essential role of bdnf in the mesolimbic dopamine pathway in social defeat stress. Science 311, 864-869.

Boer, M.C., Joosten, S.A., Ottenhoff, T.H.M., 2015. Regulatory T-cells at the interface between human host and pathogens in infectious diseases and vaccination. Front. Immunol. 6, 217. doi:

10.3389/fimmu.2015.00217

Bravo, J.A., Forsythe, P., Chew, M. V., Escaravage, E., Savignac, H.M., Dinan, T.G., Bienenstock, J., Cryan, J.F., 2011. Ingestion of Lactobacillus strain regulates emotional behavior and central GABA receptor expression in a mouse via the vagus nerve. Proc. Natl. Acad. Sci. 108, 16050-16055.

Caporaso, J.G., Kuczynski, J., Stombaugh, J., Bittinger, K., Bushman, F.D., Costello, E.K., Fierer, N., Peña, A.G., Goodrich, J.K., Gordon, J.I., Huttley, G.A., Kelley, S.T., Knights, D., Koenig, J.E., Ley, R.E., Lozupone, C.A., Mcdonald, D., Muegge, B.D., Pirrung, M., Reeder, J., Sevinsky, J.R., Turnbaugh, P.J., Walters, W.A., Widmann, J., Yatsunenko, T., Zaneveld, J., Knight, R., 2010. QIIME allows analysis of high- throughput community sequencing data. Nature Methods 7, 335 336. 


\section{Aadil Bharwani}

Clarke, G., Grenham, S., Scully, P., Fitzgerald, P., Moloney, R.D., Shanahan, F., Dinan, T.G., Cryan, J.F., 2012. The microbiome-gut-brain axis during early life regulates the hippocampal serotonergic system in a sex-dependent manner. Mol. Psychiatry. 18, 666-673.

Clarke, T.B., Davis, K.M., Lysenko, E.S., Zhou, A.Y., Yu, Y., Weiser, J.N., 2010. Recognition of peptidoglycan from the microbiota by Nod1 enhances systemic innate immunity. Nat. Med. 16, $228-231$.

Collins, S.M., Bercik, P., 2009. The relationship between intestinal microbiota and the central nervous system in normal gastrointestinal function and disease. Gastroenterology 136, 2003-2014.

Desbonnet, L., Clarke, G., Shanahan, F., Dinan, T.G., Cryan, J.F., 2014. Microbiota is essential for social development in the mouse. Mol. Psychiatry. 19, 146.

Desbonnet, L., Garrett, L., Clarke, G., Kiely, B., Cryan, J.F., Dinan, T.G., 2010. Effects of the probiotic Bifidobacterium infantis in the maternal separation model of depression. Neuroscience 170, 117988.

Dinan, T.G., 2005. Stress: the shared common component in major mental illnesses. Eur. Psychiatry 20, S326-S328.

Dinan, T.G., Quigley, E.M.M., Ahmed, S.M.M., Scully, P., O’Brien, S., O’Mahony, L., O’Mahony, S., Shanahan, F., Keeling, P.W.N., 2006. Hypothalamic-pituitary-gut axis dysregulation in irritable bowel syndrome: plasma cytokines as a potential biomarker? Gastroenterology 130, 304-311.

Erny, D., Hrabě de Angelis, A.L., Jaitin, D., Wieghofer, P., Staszewski, O., David, E., Keren-Shaul, H., Mahlakoiv, T., Jakobshagen, K., Buch, T., Schwierzeck, V., Utermöhlen, O., Chun, E., Garrett, W.S., McCoy, K.D., Diefenbach, A., Staeheli, P., Stecher, B., Amit, I., Prinz, M., 2015. Host microbiota constantly control maturation and function of microglia in the CNS. Nat. Neurosci. 18, 965-977.

Erspamer, V., 1966. Occurrence of indolealkylamines in nature, in: Erspamer, V. (Eds), 5Hydroxytryptamine and Related Indolealkylamines. Springer Berlin Heidelberg, pp. 132-181.

Everard, A., Belzer, C., Geurts, L., Ouwerkerk, J.P., Druart, C., Bindels, L.B., Guiot, Y., Derrien, M., Muccioli, G.G., Delzenne, N.M., de Vos, W.M., Cani, P.D., 2013. Cross-talk between Akkermansia muciniphila and intestinal epithelium controls diet-induced obesity. Proc. Natl. Acad. Sci.110, 9066-9071.

Forsythe, P., Sudo, N., Dinan, T., Taylor, V.H., Bienenstock, J., 2010. Mood and gut feelings. Brain. Behav. Immun. 24, 9-16.

Frost, G., Sleeth, M.L., Sahuri-Arisoylu, M., Lizarbe, B., Cerdan, S., Brody, L., Anastasovska, J., Ghourab, S., Hankir, M., Zhang, S., Carling, D., Swann, J.R., Gibson, G., Viardot, A., Morrison, D., Louise Thomas, E., Bell, J.D., 2014. The short-chain fatty acid acetate reduces appetite via a central homeostatic mechanism. Nat. Commun. 5, 3611. 


\section{Aadil Bharwani}

Galley, J.D., Yu, Z., Kumar, P., Dowd, S.E., Lyte, M., Bailey, M.T., 2015. The structures of the colonic mucosa-associated and luminal microbial communities are distinct and differentially affected by a prolonged murine stressor. Gut Microbes 5, 748-760.

Gilbert, S.F., Sapp, J., Tauber, A.I., 2012. A symbiotic view of life: we have never been individuals. Q. Rev. Biol. 87, 325-341.

Gower, J.C., 1975. Generalized procrustes analysis. Psychometrika 40, 33-51.

Groux, H., O’Garra, A., Bigler, M., Rouleau, M., Antonenko, S., de Vries, J.E., Roncarolo, M.G., 1997. A CD4 ${ }^{+} \mathrm{T}$-cell subset inhibits antigen-specific T-cell responses and prevents colitis. Nature 389, $737-742$.

Heim, C., Newport, D.J., Mletzko, T., Miller, A.H., Nemeroff, C.B., 2008. The link between childhood trauma and depression: insights from HPA axis studies in humans. Psychoneuroendocrinology 33, 693-710.

Hsiao, E.Y., McBride, S.W., Hsien, S., Sharon, G., Hyde, E.R., McCue, T., Codelli, J. a, Chow, J., Reisman, S.E., Petrosino, J.F., Patterson, P.H., Mazmanian, S.K., 2013. Microbiota modulate behavioral and physiological abnormalities associated with neurodevelopmental disorders. Cell 155, $1451-63$.

Kinsey, S.G., Bailey, M.T., Sheridan, J.F., Padgett, D.A., Avitsur, R., 2007. Repeated social defeat causes increased anxiety-like behavior and alters splenocyte function in C57BL/6 and CD-1 mice. Brain. Behav. Immun. 21, 458-466.

Klooker, T.K., Braak, B., Painter, R.C., de Rooij, S.R., van Elburg, R.M., van den Wijngaard, R.M., Roseboom, T.J., Boeckxstaens, G.E., 2009. Exposure to severe wartime conditions in early life is associated with an increased risk of irritable bowel syndrome: a population-based cohort study. Am. J. Gastroenterol. 104, 2250-2256.

Langille, M.G.I., Zaneveld, J., Caporaso, J.G., Mcdonald, D., Knights, D., Reyes, J.A., Clemente, J.C., Burkepile, D.E., Thurber, R.L.V., Knight, R., Beiko, R.G., Huttenhower, C., 2013. Predictive functional profiling of microbial communities using 16S rRNA marker gene sequences. Nat. Biotechnol. 31, 814-821.

Maes, M., 1995. Evidence for an immune response in major depression: a review and hypothesis. Prog. Neuro-Psychopharmacology Biol. Psychiatry 19, 11-38.

Mariat, D., Firmesse, O., Levenez, F., Guimarăes, V., Sokol, H., Doré, J., Corthier, G., Furet, J.-P., 2009. The Firmicutes/Bacteroidetes ratio of the human microbiota changes with age. BMC Microbiol. 9, 123.

Mayer, E.A., 2000. The neurobiology of stress and gastrointestinal disease. Gut 47, 861-869.

Muyzer, G., De Waal, E.C., Uitterlinden, A.G., 1993. Profiling of complex microbial populations by denaturing gradient gel electrophoresis analysis of polymerase chain reaction-amplified genes coding for 16S rRNA. Appl. Environ. Microbiol. 59, 695-700. 


\section{Aadil Bharwani}

Nadkarni, M.A., Martin, F.E., Jacques, N.A., Hunter, N., 2002. Determination of bacterial load by realtime PCR using a broad-range (universal) probe and primers set. Microbiology 148, 257-266.

Neufeld, K.M., Kang, N., Bienenstock, J., Foster, J.A., 2011. Reduced anxiety-like behavior and central neurochemical change in germ-free mice. Neurogastroenterol. Motil. 23, 255-265.

O’Mahony, L., McCarthy, J., Kelly, P., Hurley, G., Luo, F., Chen, K., O’Sullivan, G.C., Kiely, B., Collins, J.K., Shanahan, F., Quigley, E.M.M., 2005. Lactobacillus and bifidobacterium in irritable bowel syndrome: symptom responses and relationship to cytokine profiles. Gastroenterology 128, 541-551.

O’Mahony, S.M., Marchesi, J.R., Scully, P., Codling, C., Ceolho, A.-M., Quigley, E.M.M., Cryan, J.F., Dinan, T.G., 2009. Early life stress alters behavior, immunity, and microbiota in rats: implications for irritable bowel syndrome and psychiatric illnesses. Biol. Psychiatry 65, 263-7.

Perez-Burgos, A., Mao, Y.-K., Bienenstock, J., Kunze, W.A., 2014. The gut-brain axis rewired: adding a functional vagal nicotinic "sensory synapse". FASEB J. 28, 3064-74.

Sanderson, S., Boardman, W., Ciofi, C., Gibson, R., 2006. Human gut microbes associated with obesity. Nature 444, 1022-1023.

Schroeder, F. a, Lin, C.L., Crusio, W.E., Akbarian, S., 2007. Antidepressant-like effects of the histone deacetylase inhibitor, sodium butyrate, in the mouse. Biol. Psychiatry 62, 55-64.

Sibley, C.D., Grinwis, M.E., Field, T.R., Eshaghurshan, C.S., Faria, M.M., Dowd, S.E., Parkins, M.D., Rabin, H.R., Surette, M.G., 2011. Culture enriched molecular profiling of the cystic fibrosis airway microbiome. PLoS One 6, e22702.

Sudo, N., Chida, Y., Aiba, Y., Sonoda, J., Oyama, N., Yu, X.-N., Kubo, C., Koga, Y., 2004. Postnatal microbial colonization programs the hypothalamic-pituitary-adrenal system for stress response in mice. J. Physiol. 558, 263-75.

Thompson, J.A., Oliveira, R.A., Djukovic, A., Ubeda, C., Xavier, K.B., 2015. Manipulation of the quorum sensing signal ai-2 affects the antibiotic-treated gut microbiota. Cell Rep. 10, 1-11.

Whelan, F.J., Verschoor, C.P., Stearns, J.C., Rossi, L., Luinstra, K., Loeb, M., Smieja, M., Johnstone, J., Surette, M.G., Bowdish, D.M., 2014. The loss of topography in the microbial communities of the upper respiratory tract in the elderly. Ann. Am. Thorac. Soc. 11, 513-521.

Wikoff, W.R., Anfora, A.T., Liu, J., Schultz, P.G., Lesley, S.A., Peters, E.C., Siuzdak, G., 2009. Metabolomics analysis reveals large effects of gut microflora on mammalian blood metabolites. Proc. Natl. Acad. Sci. 106, 3698-3703.

Wyss, M.T., Magistretti, P.J., Buck, A., Weber, B., 2011. Labeled acetate as a marker of astrocytic metabolism. J. Cereb. Blood Flow Metab. 31, 1668-1674.

Yano, J.M., Yu, K., Donaldson, G.P., Shastri, G.G., Ann, P., Ma, L., Nagler, C.R., Ismagilov, R.F., Mazmanian, S.K., Hsiao, E.Y., 2015. Indigenous bacteria from the gut microbiota regulate host serotonin biosynthesis. Cell 161, 264-276. 
Aadil Bharwani

Table 1. Functional pathways with reduced predicted representation in the microbiota of mice following chronic social defeat

\begin{tabular}{ll}
\hline \multicolumn{1}{c}{ Function } & Log2 Fold Change in Frequency \\
\hline Fatty acid biosynthesis & -0.218 \\
Fatty acid metabolism & -0.214 \\
Butanoate metabolism & -0.219 \\
Propanoate metabolism & -0.221 \\
Tryptophan biosynthesis & -0.222 \\
Tyrosine biosynthesis & -0.222 \\
Tryptophan metabolism & -0.217 \\
Tyrosine metabolism & -0.218 \\
Bacterial chemotaxis & -0.227 \\
Bacterial motility proteins & -0.227 \\
Bacterial secretion system & -0.223 \\
\hline
\end{tabular}


Aadil Bharwani

Figure legends

Figure 1. Effect of chronic social defeat on behavior. (A) Percentage of time spent in the aggressor interaction zone by defeated $(n=15)$ and control mice $(n=19)$ during the aggressor interaction test. (B) and (C) Social preference and sociability scores of mice from control $(n=20)$ and defeated groups $(n=15)$ during the three-chambered sociability test. (D) Rearing behavior exhibited by defeated mice $(n=15)$ versus the control group $(n=20)$ across all time intervals on the OFT. (E) Time spent in the center of the open field for control $(n=20)$ and defeated groups $(n=15) .{ }^{* *}(P<$ 0.01), *** $(\mathrm{P}<0.001)$.

Figure 2. Microbial community diversity and profile differences between groups according to stress exposure. (A) Rarefaction curves of alpha diversity measure (Faith's PD) according to stress exposure (control versus defeated) and time point (before and after exposure to CSD). (B) Chao1 richness estimates for microbiome community in the control $(n=9)$ and defeated groups $(n=9)$. (C) PCoA plots of unweighted Unifrac distances of samples from the control (red) and defeated groups (blue). * $(\mathrm{P}<0.05)$. For interpretation of the references to color in this figure legend, the reader is referred to the web version of the article.

Figure 3. Effect of chronic social defeat on structural changes in the microbiota and association with behavioral deficits. (A) Taxonomic distribution at the phylum and class level of fecal samples derived from the control and defeated groups. (B) Statistically significant changes in OTUs belonging to either the Firmicutes or Bacteroidetes phyla, displayed as fold change in defeated mice relative to the control group. (C). Procrustes plot comparing aggressor interaction behavioral data and the microbiome profile (unweighted unifrac distances) of each mouse from control and defeated groups. Lines connect the behavioral and phylogenetic data of a specific sample. For 
Aadil Bharwani

interpretation of the references to color in this figure legend, the reader is referred to the web version of the article.

Figure 4. Effect of chronic social defeat on splenocyte phenotype and immune function. (A) IL-6 levels in the serum of defeated $(n=9)$ and unstressed control mice $(n=10)$. (B) Foxp3+CD4+ CD25+ splenocyte levels from control $(n=10)$ or defeated groups $(n=8)$ following exposure to CSD. (C) IL$10+C D 4+C D 25+$ expression in the spleens of control $(n=10)$ and defeated mice $(n=8)$. (D) In vitro IL-10 release following stimulation of splenocytes with anti-CD3/CD28 antibodies; defeated group $(n=8)$, control group $(n=10) .(E)$ Levels of CD80+CD11c+ splenocytes in control $(n=10)$ and defeated mice (n=7). (F) Gating strategy for IL-10+ CD4+ CD25+ splenocytes. * $(\mathrm{P}<0.05), * *(\mathrm{P}<$ 0.01), and ${ }^{* * *}(\mathrm{P}<0.001)$.

Figure 5. Effect of chronic social defeat on the predicted functional metagenome profile following CSD. (A) Predicted functional diversity using the Shannon index of the microbial communities in control $(n=9)$ and defeated mice $(n=9)$. (B) PCoA plots of Bray-Curtis distances of the predicted functional profile of KEGG pathways in control (red) and defeated groups (blue). ${ }^{*}(\mathrm{P}<0.05)$. For interpretation of the references to color in this figure legend, the reader is referred to the web version of the article. 
Figure 1
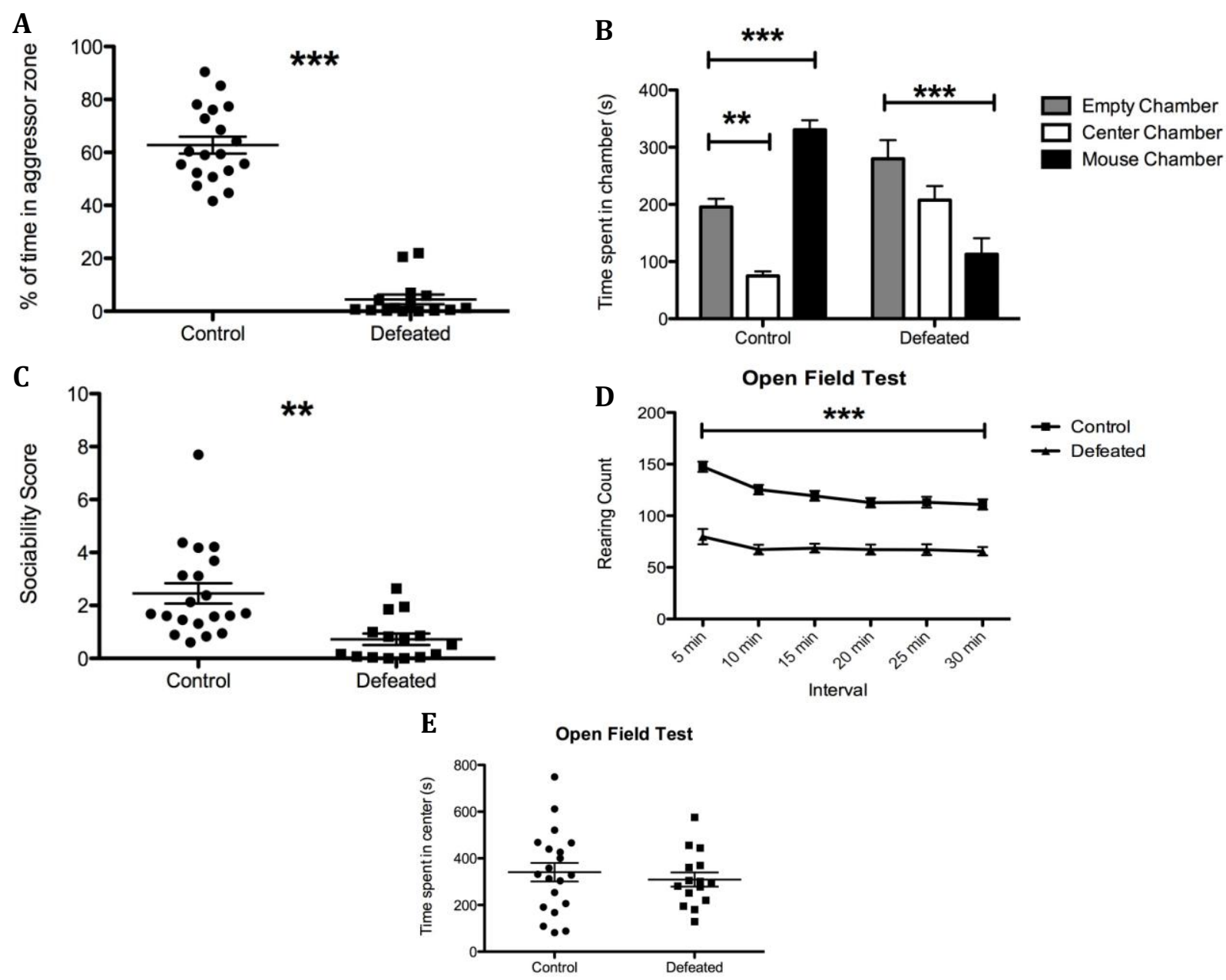
Figure 2
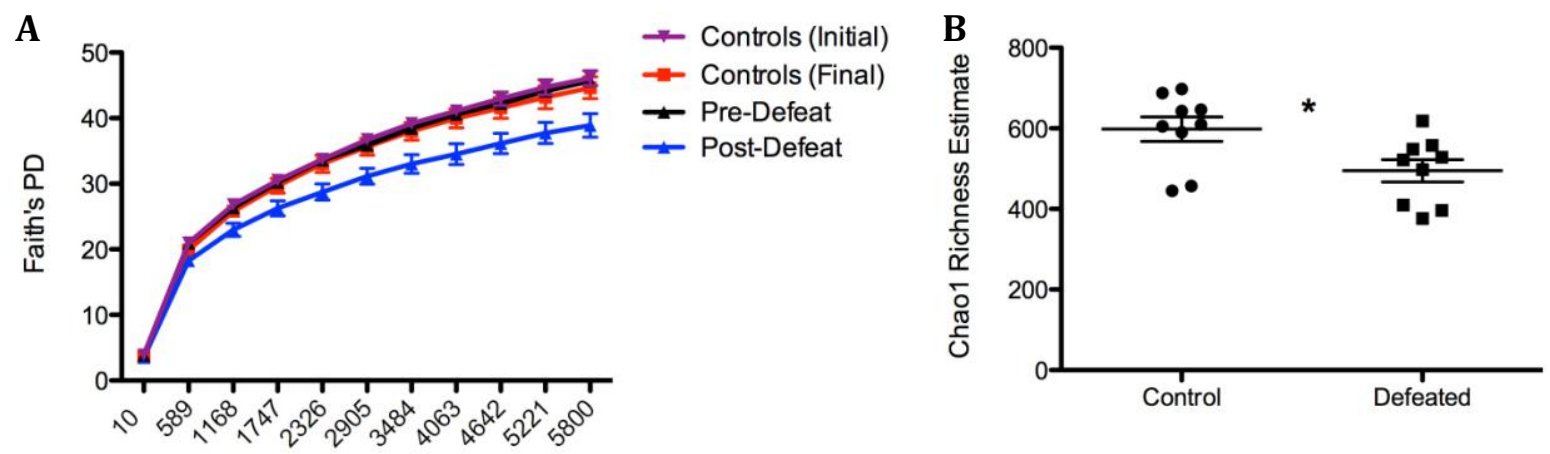

Sequences Per Sample

C

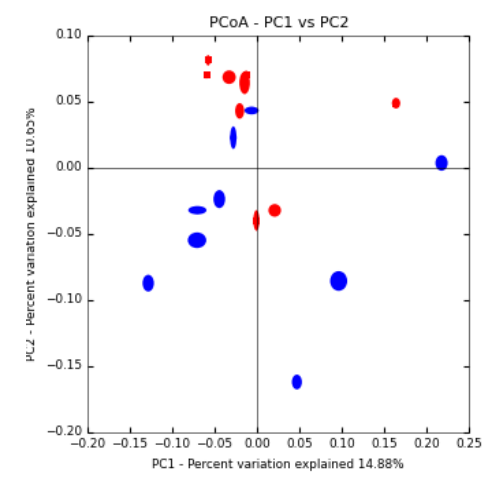

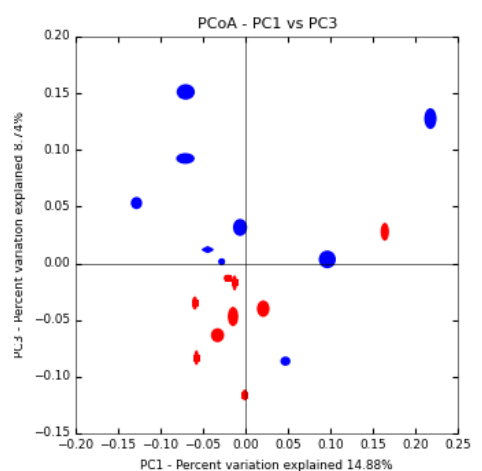

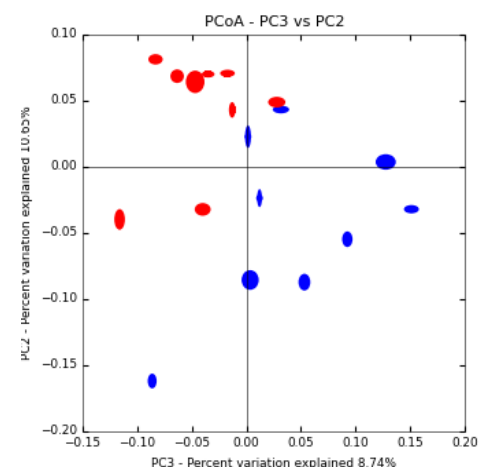


Figure 3
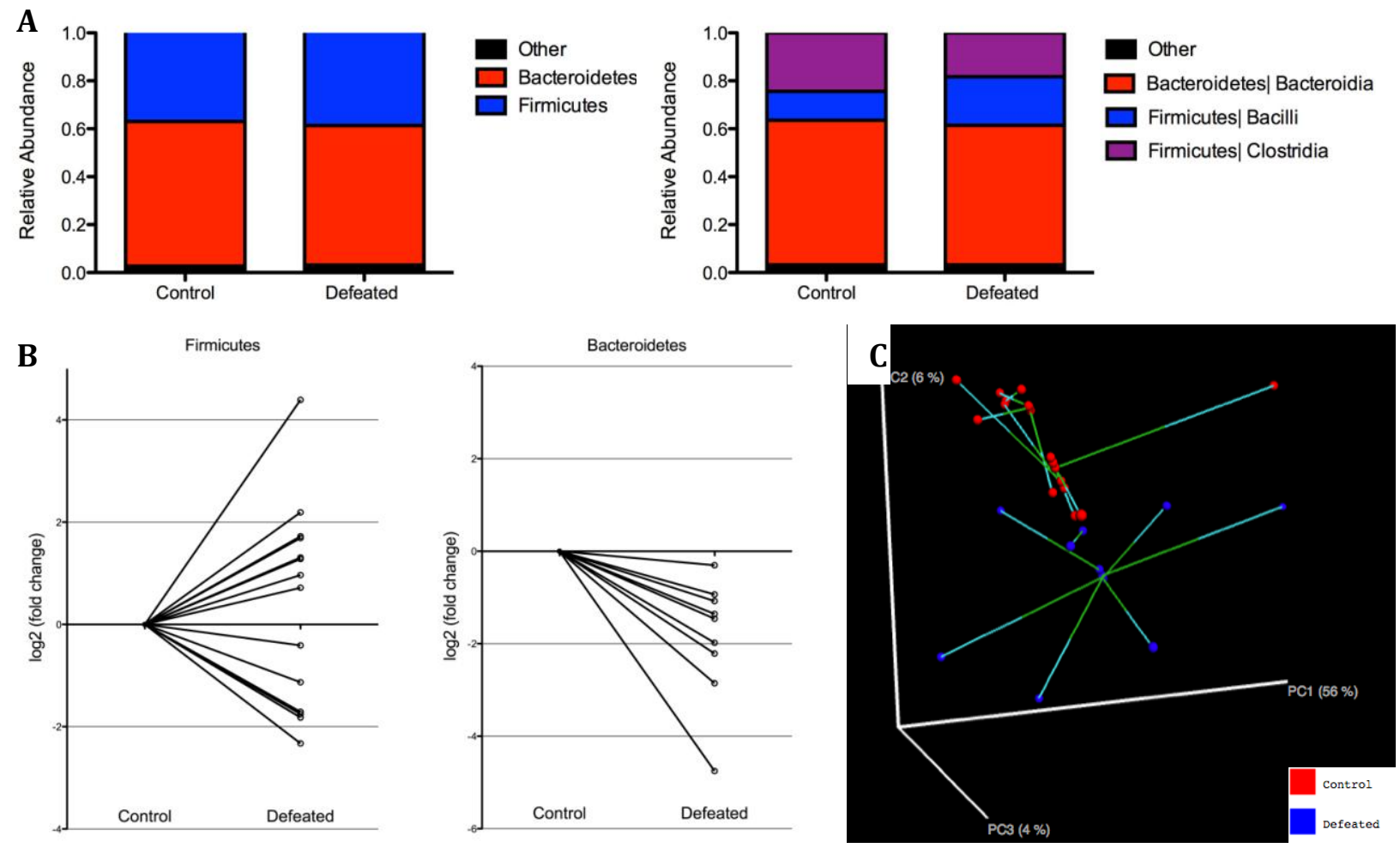
Figure 4

A IL-6 Levels (5 days post-stressor)

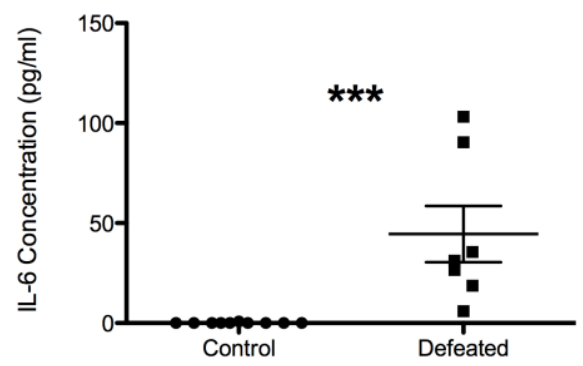

C

IL-10+ CD4+ CD25+ (17 days post-stressor)

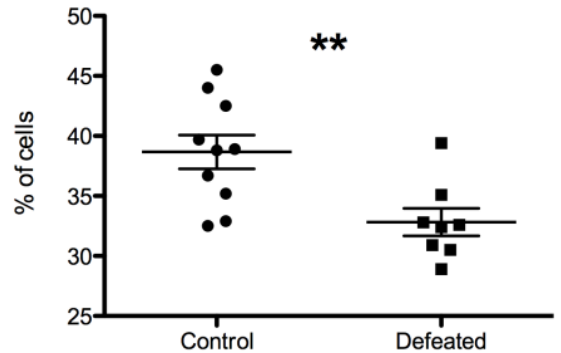

E

CD80+ CD11C+ (5 days post-stressor)

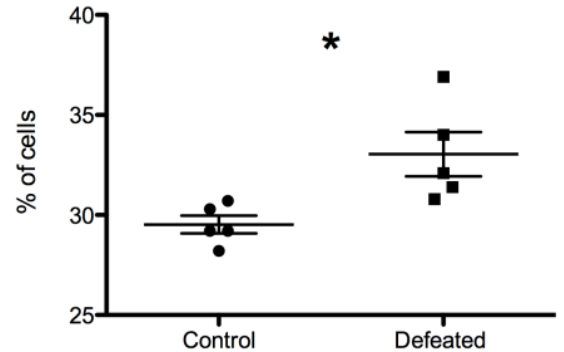

B IL-10+CD4+ CD25+ (5 days post-stressor)

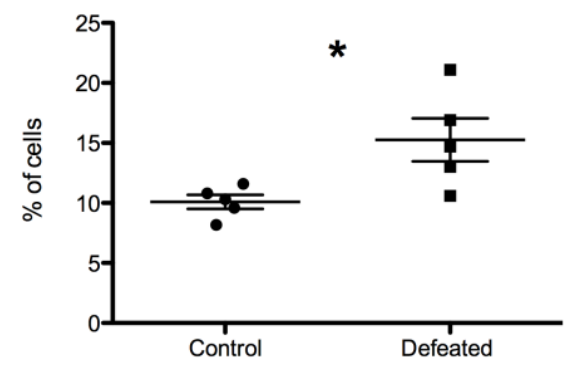

D

anti-CD3/CD28 Stimulation (17 days post-stressor)

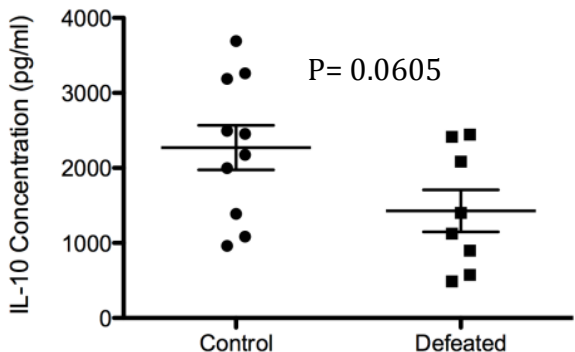

F

CD80+ CD11C+ (17 days post-stressor)

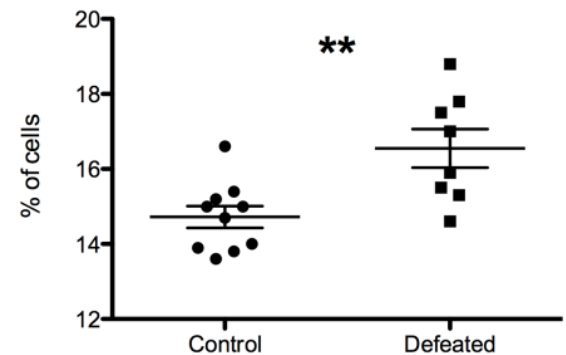


Figure 5

A

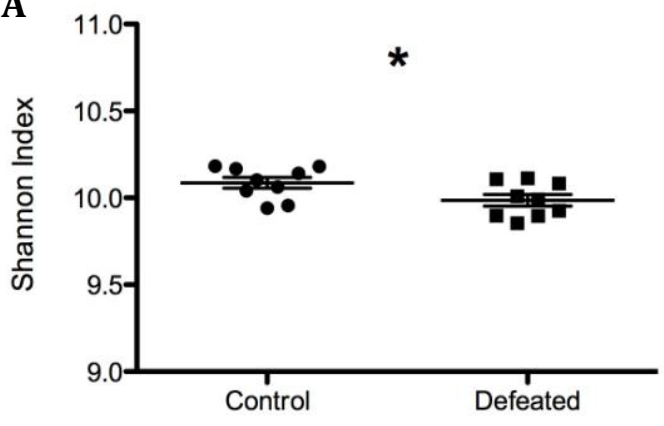

B
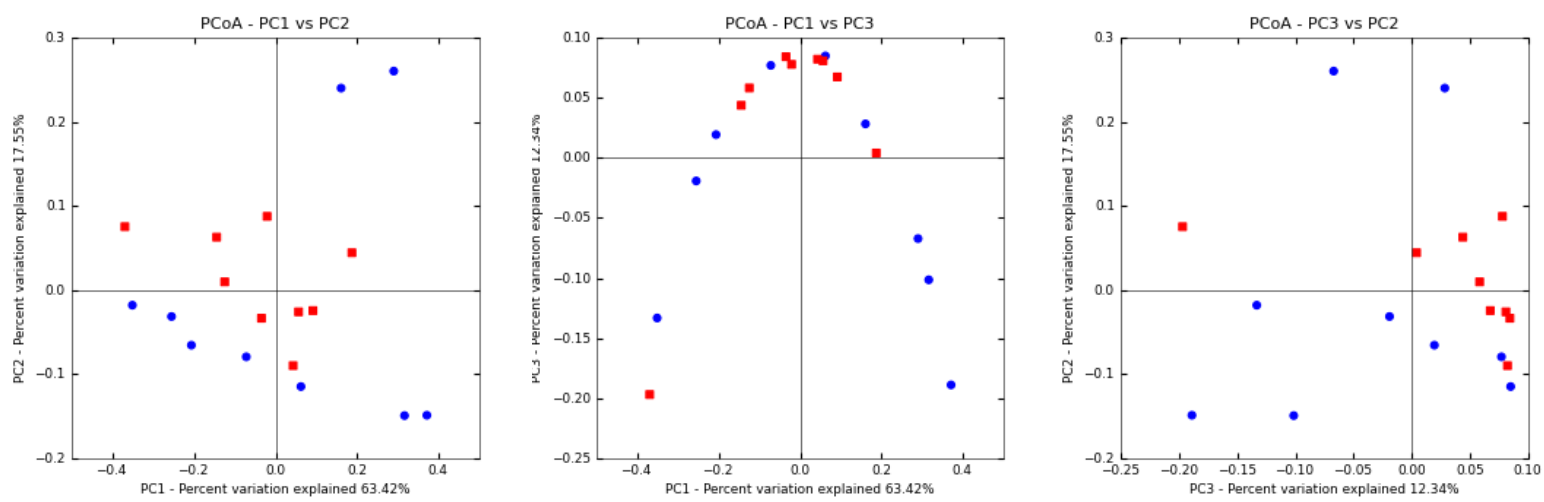